\title{
Delaying skeletal-related events in a randomized phase 3 study of denosumab versus zoledronic acid in patients with advanced cancer: an analysis of data from patients with solid tumors
}

\author{
David Henry • Saroj Vadhan-Raj • Vera Hirsh • Roger von Moos • \\ Vania Hungria • Luis Costa • Penella J Woll • Giorgio Scagliotti • \\ Geoffrey Smith • Amy Feng • Susie Jun • Roger Dansey • Howard Yeh
}

Received: 1 March 2013 / Accepted: 8 October 2013 / Published online: 26 October 2013

(C) Springer-Verlag Berlin Heidelberg 2013

\begin{abstract}
Purpose Bone complications of metastatic disease, including skeletal-related events (SREs), impair patients' functioning and quality of life. In a randomized, phase 3 trial of 1,776 patients with metastases from solid tumors (except breast or prostate) or multiple myeloma, denosumab was non-inferior to zoledronic
\end{abstract}

Trial Registration: This study is registered with ClinicalTrials.gov with the identifier NCT00330759.

\author{
D. Henry $(\bowtie)$ \\ Joan Karnell Cancer Center, Pennsylvania Hospital, \\ 230 W Washington Square, Philadelphia, PA 19106, USA \\ e-mail: davidhenry@pennoncology.com \\ S. Vadhan-Raj \\ M.D. Anderson Cancer Center, University of Texas, Houston, TX, \\ USA \\ V. Hirsh \\ McGill University Health Center, Montreal, QC, Canada \\ R. von Moos \\ Kantonsspital Graubünden, Chur, Switzerland \\ V. Hungria \\ Irmandade da Santa Casa de Misericordia de São Paulo, São Paulo, \\ Brazil \\ L. Costa \\ Hospital de Santa Maria and Instituto de Medicina Molecular, \\ Lisboa, Portugal \\ P. J. Woll \\ University of Sheffield, Sheffield, UK \\ G. Scagliotti \\ University of Torino, Orbassano, Italy \\ G. Smith $\cdot$ A. Feng $\cdot$ S. Jun $\cdot$ R. Dansey $\cdot$ H. Yeh \\ Amgen Inc., Thousand Oaks, CA, USA
}

acid (ZA) in delaying or preventing SREs. This ad hoc analysis reports outcomes in the subgroup of 1,597 patients with solid tumors, excluding patients with multiple myeloma.

Methods Patients received monthly subcutaneous denosumab $120 \mathrm{mg}$ or intravenous ZA $4 \mathrm{mg}$, adjusted for creatinine clearance, with calcium and vitamin D supplementation recommended. Endpoints included times to first on-study SRE, first-and-subsequent SREs, and pain worsening.

Results Denosumab significantly delayed time to first onstudy SRE compared with ZA (HR, 0.81; $95 \%$ CI, 0.68 0.96 ) and time to first-and-subsequent SREs (RR, 0.85; $95 \%$ CI, 0.72-1.00). Denosumab also significantly delayed time to development of moderate or severe pain (HR, $0.81 ; 95 \% \mathrm{CI}$, 0.66-1.00), pain worsening (HR, 0.83; $95 \% \mathrm{CI}, 0.71-0.97$ ), and worsening pain interference in patients with no/mild baseline pain (HR, 0.77; $95 \%$ CI, 0.61-0.96). Adverse event rates were $96 \%$ in both groups. Grade 3 or 4 hypocalcemia, mostly without clinical sequelae, was more frequent in denosumab-treated patients (denosumab $4 \%$, ZA $2 \%$ ). Osteonecrosis of the jaw occurred infrequently (denosumab $0.8 \%$, ZA $1.1 \%$ ).

Conclusions Denosumab was more effective in delaying or preventing SREs in patients with bone metastases from solid tumors and also prevented pain progression compared to ZA in this ad hoc analysis.

Keywords Denosumab · Bone resorption · Bone metastases · Cancer-related pain

\section{Introduction}

Bone is a common site for tumor spread in patients with solid tumors and patients with bone metastases are at risk for 
developing skeletal complications of their disease [1] driven through pathological activation of osteoclasts by tumor cells in the bone. Skeletal-related events (SREs), which include pathological fractures, a need for orthopedic surgery and radiotherapy to prevent or repair major structural damage, and spinal cord and nerve root compression, represent a significant cause of morbidity including loss of mobility and functional impairments $[2,3]$. In addition, bone pain is the most prevalent of all cancer-associated types of pain and may be severe, necessitating opiate analgesics and palliative radiation therapy, which are often accompanied by a substantial decline in patient-reported quality of life (QOL) [4]. SREs in patients with metastatic disease are also associated with poor prognosis and reduced survival [5-7].

Intravenous (IV) bisphosphonates have been the mainstay of treatment for the prevention of SREs in patients with metastatic solid tumors. In the pivotal phase 3 trial, zoledronic acid (ZA) demonstrated efficacy in preventing skeletal complications in patients with bone metastases secondary to solid tumors other than breast or prostate [8]. In that trial, ZA $4 \mathrm{mg}$ was compared with placebo in 773 patients with bone metastases from solid tumors other than breast or prostate. Patients with non-small cell lung cancer (NSCLC) comprised $50 \%$ of the total and more than 20 other tumor types were represented including renal cell carcinoma, colorectal cancer, small cell lung cancer, and bladder cancer. Patients received a median of four infusions of ZA, which provided a longer median time to first SRE (236 versus 155 days for placebo; $p=0.009)$ and a lower risk for developing multiple SREs compared with placebo $(\mathrm{HR}=0.693 ; p=0.003)$.

Despite antiresorptive treatment, SREs may still occur. For patients with renal insufficiency in particular, restrictions on ZA use in this population may limit full treatment benefit [8]. Per Zometa ${ }^{\circledR}$ (Novartis, East Hanover, NJ, USA) prescribing information [9], ZA is not indicated for patients with creatinine clearance $<30 \mathrm{~mL} / \mathrm{min}$ and requires either dose adjustment in patients with creatinine clearance $<60 \mathrm{~mL} / \mathrm{min}$ or dose withholding if creatinine levels rise during treatment to minimize the risk for renal injury. Additionally, IV ZA therapy requires specialized administration settings and has been associated with occurrence of an acute flu-like syndrome, particularly after the first dose. Alternate therapeutic options are therefore needed to further reduce the occurrence of SREs, while minimizing potential toxicities and allowing more patients with bone metastases to receive treatment.

Denosumab (XGEVA ${ }^{\circledR}$ Amgen, Thousand Oaks, CA, USA) is a subcutaneously dosed monoclonal antibody against RANK ligand (RANKL) for prevention of SREs in patients with solid tumors metastatic to bone [10]. Findings from both preclinical studies and trials of patients with bone metastases suggest that tumor-induced bone destruction is largely caused by activation of bone-resorbing osteoclasts [11]. RANKL, the key activator of osteoclasts, is the main mediator of this bone resorption through tumor-secreted growth factors and cytokines [12]. Three international, randomized, double blind, double dummy phase 3 studies have evaluated denosumab versus ZA for the delay and treatment of SREs in breast and prostate cancers, and in combined solid tumors (non-prostate, non-breast) and multiple myeloma [13-15]. Denosumab's superior efficacy over ZA was demonstrated in the studies of patients with advanced breast or prostate cancer, as well as in a prespecified integrated analysis of all patients enrolled across the three studies $[13,14,16]$. In the study comparing denosumab with ZA in the prevention of SREs in patients with various solid tumors or multiple myeloma [15], denosumab was overall non-inferior to ZA and demonstrated a $16 \%$ reduction in risk for the primary endpoint of time to first on-study SRE, with a median time to first SRE of 20.6 months for denosumab and 16.3 months for ZA. In order to provide an analysis comparable to the previous registrational study of ZA in patients with solid tumors, we report here data from an ad hoc analysis for the solid tumor subset of this study, excluding the multiple myeloma cohort, which represented $10 \%$ of the study population. Together with results already reported from the two phase 3 studies in prostate and breast cancers $[13,14]$, these results allow comparison of the efficacy and safety of denosumab versus ZA in all patients with solid tumors.

\section{Methods}

Adults with solid tumors (excluding breast and prostate cancers) were eligible for this study if they had radiographic evidence of at least one bone metastasis (osteolytic or osteoblastic [17]), Eastern Cooperative Oncology Group (ECOG) performance status $\leq 2$, adequate organ function, and a life expectancy of at least 6 months. Informed written consent was also required. Patients with multiple myeloma, while included in the previously published full study results [15], are not included in this ad hoc analysis of patients with solid tumors.

Patients who had current or prior IV bisphosphonate use, current or prior oral bisphosphonate use for cancer-related bone disease, planned radiation or surgery to bone, any prior use of denosumab, a prior history of osteonecrosis/osteomyelitis of the jaw (ONJ), or a planned or nonhealed oral surgery or invasive dental procedure were ineligible.

Patients were randomized 1:1 using an interactive voice response system to receive either subcutaneous (SC) denosumab $120 \mathrm{mg}$ and IV placebo, or SC placebo and IV ZA $4 \mathrm{mg}$ (or equivalent creatinine clearance-adjusted dose in patients with baseline creatinine clearance $\leq 60 \mathrm{~mL} / \mathrm{min}$ ) [9] every 4 weeks. Patients were also strongly advised to take supplemental calcium $\geq 500 \mathrm{mg}$ and vitamin $\mathrm{D} \geq 400 \mathrm{IU}$. Randomization was stratified by tumor type, previous SRE, 
systemic anti-neoplastic therapy, and geographic region. Enrollment of patients with NSCLC was limited to $60 \%$ of the total study number. Investigators, patients, and study personnel were blinded to treatment assignments.

The main outcomes of this analysis were time to first onstudy SRE and time to first-and-subsequent SRE (multipleevent analysis) assessed by superiority tests comparing the effects of denosumab versus ZA [15]. SREs were defined as pathologic fractures (vertebral or nonvertebral), radiation therapy to bone (including the use of radioisotopes), surgery to bone, or spinal cord compression. Fractures were assessed by skeletal surveys (X-rays) every 12 weeks as well as by radiographic assessments (X-ray, computed tomography, or magnetic resonance imaging) performed as part of standard care and were identified or confirmed by independent, blinded central radiology review. Spinal cord compression events were also confirmed by blinded central radiology review. Only events that occurred $\geq 21$ days after the previous event were counted as an SRE [15]. Overall disease progression was assessed by the investigators for all patients on study and overall survival was reported by the investigators for all patients on study and in survival follow-up.

In order to compare the relative benefits of the two treatments, the number needed to treat (NNT) for denosumab compared with ZA was calculated as the inverse of the difference of the annualized or patient-year adjusted rates between the two treatments. In this event-driven trial, patients were on study for different lengths of time; therefore, the use of patient-year adjusted rates most accurately describes treatment benefit regardless of duration.

The 11-point Brief Pain Inventory-Short Form (BPI-SF) was used to assess development of moderate or severe pain on study as well as time to pain worsening or improvement [18]. Pain was categorized as no pain ( 0 points), mild pain (1-4 points), moderate pain (5-6 points), or severe pain (7-10 points). Analgesic use was assessed through week 45 of treatment using the eight-point Analgesic Quantification Algorithm (AQA). AQA ratings of 0 or 1 indicate no analgesic use or use of nonopioid analgesics, respectively, and a rating of 2 indicates use of weak opioids (e.g., meperidine, codeine, or tramadol). AQA ratings of 3 through 7 indicate use of strong opioids in daily morphine-equivalent doses ranging from $\leq 75$ to $>600 \mathrm{mg}$ [19]. The BPI-SF was also used to assess pain interference on an 11-point scale $(0=$ does not interfere; $10=$ completely interferes). The BPI-SF for pain interference includes seven items: general activity, mood, walking ability, work, relations with others, sleep, and enjoyment of life [18].

Potential AEs of ONJ were identified through review of case report forms and adverse event listings using a predefined list of Medical Dictionary for Regulatory Activities (MedDRA v 12.0) preferred terms. Each event was independently confirmed by a blinded ONJ Adjudication Committee consisting of a panel of expert external reviewers.
Detailed statistical methods for the study can be found in Henry et al. [15]. Briefly, the time to first on-study SRE, time to overall survival, and time to disease progression were analyzed using a Cox proportional hazards model stratified by the randomization-stratification factors and including treatment groups, age, gender, time from primary diagnosis of primary cancer to first evidence of metastatic disease, time from initial diagnosis to first bone metastasis, visceral metastasis, and baseline ECOG performance status as independent variables. Time to first-and-subsequent on-study SRE (multiple event analysis) was analyzed using the Anderson and Gill approach. Efficacy analyses were based on the intent-to-treat principle. Safety analyses included all patients who received at least one dose of study medication.

\section{Results}

This international, randomized, double blind, double dummy study was conducted at 321 centers between June 212006 and April 302009 (the primary analysis cutoff date) according to the Declaration of Helsinki and the International Conference on Harmonisation Tripartite Guideline on Good Clinical Practice. Patients provided informed consent prior to treatment and oversight was provided by the independent ethics committee or institutional review board at each study center.

The solid tumor analysis included 1,597 patients. Overall, $17 \%$ of patients remained on study at the time of the primary analysis data cutoff date (Fig. 1). The median (Q1, Q3) time on study was $6.7(3.2,13.0)$ months for denosumab and 6.4 $(3.1,12.9)$ months for ZA. Patients in the denosumab group received a median $(\mathrm{Q} 1, \mathrm{Q} 3)$ of $7.0(3.0,13.0) \mathrm{SC}$ injections; patients in the ZA group received a median $(\mathrm{Q} 1, \mathrm{Q} 3)$ of 6.0 $(3.0,12.0) \mathrm{IV}$ infusions. The most common medical reasons

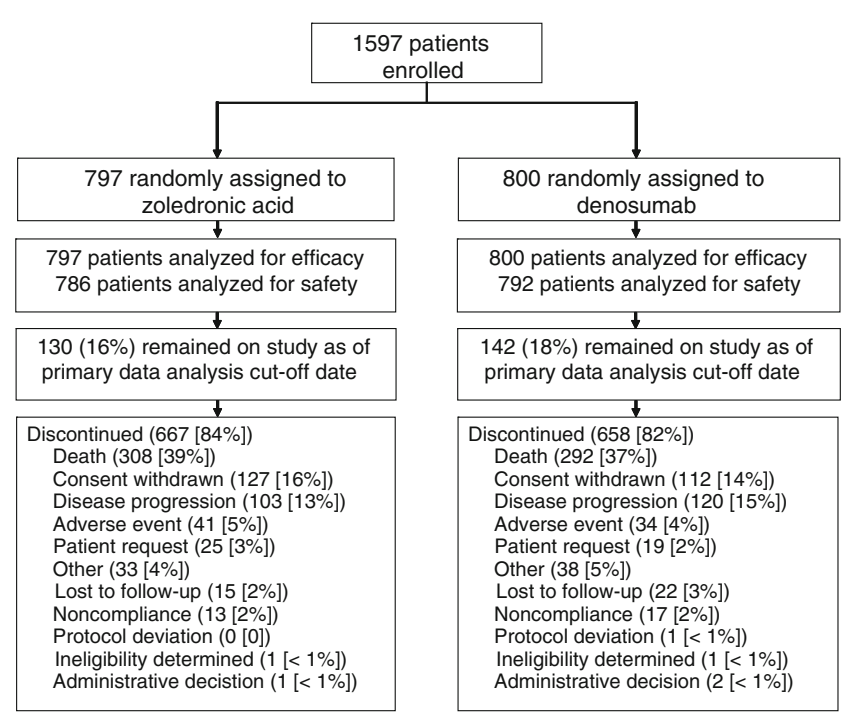

Fig. 1 Patient disposition 
for study discontinuation were death $(38 \%)$ and disease progression (14\%).

Demographics and baseline characteristics

Between patients randomized to the denosumab $(N=800)$ and ZA $(N=797)$ treatment groups, baseline characteristics were relatively balanced (Table 1 ). Approximately $64 \%$ were male and approximately $88 \%$ were Caucasian.

NSCLC was the primary tumor type in 702 (44\%) patients. The most common other primary tumor types included renal cell carcinoma ( $n=155 ; 10 \%)$, small cell lung cancer ( $n=109 ; 7 \%)$, bladder $(n=63 ; 4 \%)$, rectal $(n=60 ; 4 \%)$, and colon $(n=59 ; 4 \%)$ cancers. Overall, 917 (57\%) patients had visceral metastases at baseline. The median (Q1, Q3) time

Table 1 Baseline demographics and disease characteristics

\begin{tabular}{|c|c|c|}
\hline $\begin{array}{l}\text { Characteristics } \\
n(\%) \text { or median }\end{array}$ & $\begin{array}{l}\text { Denosumab } \\
(N=800)\end{array}$ & $\begin{array}{l}\text { Zoledronic acid } \\
(N=797)\end{array}$ \\
\hline \multicolumn{3}{|l|}{ Sex } \\
\hline Female & $269(34)$ & $299(38)$ \\
\hline Male & $531(66)$ & $498(62)$ \\
\hline \multicolumn{3}{|l|}{ Race/ethnicity } \\
\hline White or Caucasian & $702(88)$ & $697(87)$ \\
\hline Asian or Japanese & $32(4)$ & $40(5)$ \\
\hline Hispanic or Latino & $43(5)$ & $31(4)$ \\
\hline Black or African American & $17(2)$ & $22(3)$ \\
\hline Other & $6(1)$ & $7(1)$ \\
\hline Age, years, median (min, max) & $59(18,89)$ & $61(22,87)$ \\
\hline ECOG performance status of 0 or 1 & $678(85)$ & $654(82)$ \\
\hline Previous SRE & $379(47)$ & $381(48)$ \\
\hline \multicolumn{3}{|l|}{ Primary tumor types ${ }^{\mathrm{a}}$} \\
\hline Non-small cell lung cancer & $350(44)$ & $352(44)$ \\
\hline Other & $448(56)$ & $445(56)$ \\
\hline $\begin{array}{l}\text { Months from initial diagnosis } \\
\text { of bone metastasis to } \\
\text { randomization, median } \\
(\mathrm{Q} 1, \mathrm{Q} 3)\end{array}$ & $1.8(0.9,3.8)$ & $1.8(0.9,3.9)$ \\
\hline Visceral metastases & $471(59)$ & $446(56)$ \\
\hline Liver & $170(21)$ & $166(21)$ \\
\hline Lung & $237(30)$ & $162(20)$ \\
\hline Other & $317(40)$ & $338(42)$ \\
\hline $\begin{array}{l}\text { Pain at baseline (BPI-SF score; } \\
\text { range } 0 \text { to } 10) \\
\text { Worst pain, median score (Q1, Q3) }\end{array}$ & $5.0(3.0,7.0)$ & $5.0(3.0,8.0)$ \\
\hline No pain (0) & $74(9)$ & $72(9)$ \\
\hline Mild pain (1 to 4 ) & $249(31)$ & $201(25)$ \\
\hline Moderate pain (5 to 6 ) & $180(22)$ & $194(24)$ \\
\hline Severe pain (7 to 10$)$ & $247(31)$ & $278(35)$ \\
\hline Missing data & $50(6)$ & $52(6)$ \\
\hline
\end{tabular}

${ }^{a}$ Percentages based on randomization from diagnosis to first metastasis was $3.0(0.0,15.8)$ months for the denosumab group and $3.5(0.1,16.3)$ months for the ZA group.

Eighty-four percent of patients in each of the denosumab $(n=674)$ and ZA $(n=671)$ groups had received prior systemic anticancer therapy. Previous SREs were reported for 379 (47\%) denosumab patients and 381 (48\%) ZA patients. Median (Q1, Q3) BPI-SF worst pain scores at baseline were 5.0 for each group $(\mathrm{Q} 1, \mathrm{Q} 3=[3.0,7.0]$ denosumab, $[3.0,8.0]$ ZA). At baseline, 502 (63\%) patients in the denosumab group and $497(62 \%)$ in the ZA group had no/low analgesic use (AQA rating 0-2).

\section{Efficacy}

The Kaplan-Meier (KM) estimate of median (95\% CI) time to first on-study SRE was 21.4 (15.7, upper estimate not reached) months in patients receiving denosumab and 15.4 $(11.9,19.2)$ months in patients receiving ZA (HR, 0.81; $95 \%$ CI, 0.68-0.96: $p=0.017$ ) (Fig. 2a), a difference of 6 months, representing a $19 \%$ reduction in hazard. The estimated risk reduction for multiple SREs was $15 \%$ in the denosumab group (328 events) compared with ZA (374 events) (RR, 0.85; $95 \%$ CI, 0.72-1.00: $p=0.048$ ) (Fig. 2b). The NNT with denosumab compared with ZA was 7.8 patient-years, indicating that treatment of 7.8 patients for 1 year with denosumab instead of ZA would prevent 1 additional SRE. The treatment effect of denosumab was consistent across various tumor types (Table 2). The most frequent type of initial SRE reported was [denosumab, ZA; $n$ (\%)] radiation to bone [111 (14\%), $137(17 \%)]$, followed by pathologic fracture [92 (12\%), $103(13 \%)]$, surgery to bone [12 (2\%), 17 (2\%)], and spinal cord compression [21 (3\%), 20 (3\%)].

In patients with no or mild baseline worst pain (BPI-SF $\leq 4)$, the median $(95 \% \mathrm{CI}) \mathrm{KM}$ estimate of time to a $>4$-point increase in BPI-SF worst pain score was $4.7(3.7,5.8)$ months in the denosumab group and $3.7(2.8,3.9)$ months in the ZA group (HR, 0.81; $95 \%$ CI, 0.66-1.00: $p=0.050$ ) (Fig. 3a). In all patients without severe worst pain at baseline (BPI-SF $\leq 8$ ), the median $(95 \% \mathrm{CI})$ time to a $\geq 2$-point increase in BPI-SF score was $5.6(4.7,6.5)$ months in the denosumab group and $4.6(3.9,5.6)$ months in the ZA group (HR, $0.83 ; 95 \% \mathrm{CI}$, 0.71-0.97: $p=0.016$ ) (Fig. 3b). In patients with a baseline pain score $\geq 2$, median time to clinically meaningful improvement in pain ( $\geq 2$-point decrease in BPI-SF worst pain score) was 2.8 months for both the denosumab and ZA groups (HR, 1.06; $95 \%$ CI, 0.92-1.23: $p=0.422$ ).

Denosumab also delayed worsening of pain interference in patients with no/mild worst pain at baseline. The median (95\% CI) KM estimate of time to a $\geq 2$-point increase in BPI-SF pain interference score was $8.2(5.6,9.6)$ months in the denosumab group and $4.8(3.7,7.5)$ months in the ZA group (HR, 0.77; $95 \% \mathrm{CI}, 0.61-0.96: p=0.021$ ). In all 


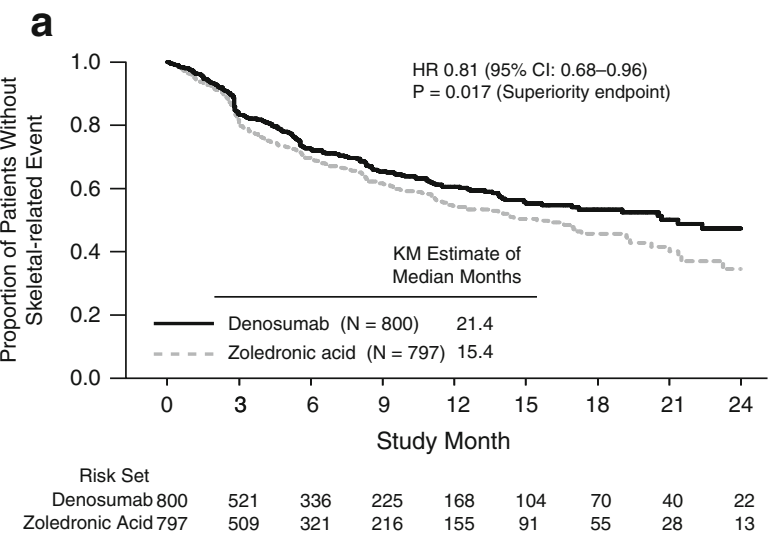

b

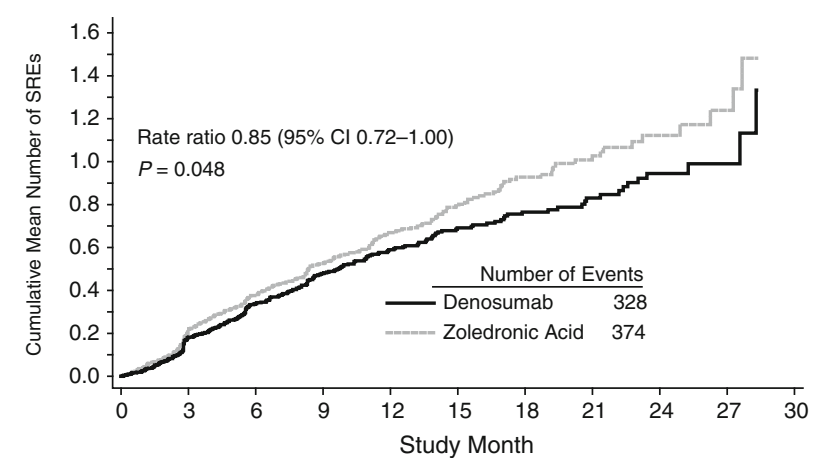

Fig. 2 Kaplan-Meier estimates of a time to first skeletal-related event (SRE) and $\mathbf{b}$ cumulative mean number of SREs. $N=$ number of patients randomized (solid tumor subset). $H R=$ hazard ratio; $C I=$ confidence interval; $K M=$ Kaplan-Meier. For the multiple events analysis (b), only events occurring $\geq 21$ days after the previous event are counted. Curves are displayed up to the last event time

patients without severe pain interference at baseline (BPI-SF $\leq 8)$, the median $(95 \% \mathrm{CI})$ time to $\mathrm{a} \geq 2$-point increase in BPISF pain interference score was $8.2(6.6,9.3)$ months in the denosumab group and $7.5(5.8,10.2)$ months in the ZA group (HR, 0.89; $95 \%$ CI, 0.76-1.04: $p=0.153$ ).

Among patients with no/low baseline analgesic use, fewer patients receiving denosumab than those receiving ZA were using strong opioids at each time point measured. The differences were statistically significant at week 13 (denosumab, $8.8 \%$; ZA, $13.0 \%$ : $p=0.047$ ), week 17 (denosumab, 9.2 \%; $\mathrm{ZA}, 16.6 \%$ : $p=0.002$ ), and week 21 (denosumab, $11.0 \%$; ZA, $18.5 \%: p=0.004)$.

Overall survival was similar in both groups. The median (95\% CI) KM estimate was $10.7(9.3,11.9)$ months for denosumab-treated patients and $10.0(8.7,11.5)$ months for ZA-treated patients (HR, 0.92; $95 \% \mathrm{CI}, 0.81-1.05: p=0.215$ ). Similarly, there was no difference between groups in time to disease progression. The median $(95 \% \mathrm{CI}) \mathrm{KM}$ estimate was $5.3(4.9,5.7)$ months for denosumab-treated and $5.4(4.8,5.7)$ months for ZA-treated patients (HR, 0.96; $95 \%$ CI, 0.85-1.08: $p=0.497)$.
The median $(\mathrm{Q} 1, \mathrm{Q} 3)$ percent decrease from baseline at week 13 for the bone turnover marker uNTx was $76.6 \%$ $(56.3,87.9 \%)$ in the denosumab group compared with $65.1 \%(34.9,80.6 \%)$ in the ZA group $(p<0.0001)$.

Drug exposure and adjustments for renal function

Because of the potential for nephrotoxicity with ZA administration, 135 (17.2\%) patients' initial IV ZA doses were adjusted per protocol in accordance with the ZA prescribing information. In addition, 65 (8.3\%) ZA patients required withholding of IV ZA dose on study for elevated serum creatinine (261 total doses withheld). No dose adjustment was required for the subcutaneously administered product.

\section{Safety}

Adverse events reported on study were relatively balanced between treatment groups. Overall, the rates of AEs occurring on study were similar (Table 3). The most common AE was anemia occurring in $30 \%$ of patients. Grade 3 or 4 hypocalcemia (corrected serum calcium of $<7.0$ to 6.0 or $<6.0 \mathrm{mg} / \mathrm{dL}$, respectively) [21], the majority of which were asymptomatic, occurred in $35(4 \%)$ and $14(2 \%)$ patients in the denosumab and ZA groups, respectively. Acute phase reactions occurring within the first 3 days of treatment were more frequent in patients receiving ZA [116 (15\%)] compared with denosumab [56 (7\%)]. Despite dose adjustment for creatinine clearance and increased serum creatinine on study, AEs associated with renal toxicity occurred more often in patients receiving ZA [81 (10\%)] compared with denosumab [56 (7\%)]. At the primary analysis cutoff date, six $(0.8 \%)$ patients in the denosumab and nine $(1.1 \%)$ in the ZA groups had experienced positively adjudicated AEs of ONJ $(p=0.45)$. Of these, three patients in the denosumab and two patients in the ZA group had resolution of their ONJ as of June 2013. A summary of patient incidence of AEs by treatment group is shown in Table 3.

\section{Discussion}

Historically, ZA established a standard of care for a bonetargeted antiresorptive therapy by demonstrating a median increase of 2 months to first SRE compared with placebo for patients with metastases from solid tumors other than breast and prostate [8]. In this analysis conducted in a similar patient population, denosumab extended the time to first SRE by a median of 6 months compared to ZA, resulting in a relative risk reduction for a first SRE of $19 \%$. The HR in delaying time to first on-study SRE is comparable to that seen in other pivotal phase 3 trials of denosumab, which showed an $18 \%$ relative reduction in the risk of first SRE in patients with 
Table 2 Outcomes for most common tumor types

$N$ number of patients randomized, Pt est point estimate, $S R E$ skeletal-related event

${ }^{a}$ Data from subgroup analysis of patients with genitourinary cancers enrolled in pivotal phase 3 studies and includes renal, bladder, and transitional cell cancers [20]

${ }^{\mathrm{b}}$ Subgroup analysis of patients per randomization with non-small cell lung cancer or small cell lung cancer in present study; data not adjusted for stratification factors

${ }^{\mathrm{c}}$ Subgroup analysis of patients per actual strata.

\begin{tabular}{|c|c|c|c|c|c|c|}
\hline & \multicolumn{3}{|c|}{ Time to first on-study SRE } & \multicolumn{3}{|c|}{$\begin{array}{l}\text { Time to first-and-subsequent } \\
\text { SRE }\end{array}$} \\
\hline & \multicolumn{3}{|c|}{ Hazard ratio } & \multicolumn{3}{|c|}{ Rate ratio } \\
\hline & Pt Est & $(95 \% \mathrm{CI})$ & $p$ value & Pt Est & $(95 \% \mathrm{CI})$ & $p$ value \\
\hline \multicolumn{7}{|l|}{ Non-prostate GU cancers ${ }^{\mathrm{a}}$} \\
\hline \multicolumn{7}{|l|}{ Zoledronic acid 4 mg Q4W $(N=125)$} \\
\hline Denosumab 120 mg Q4W $(N=102)$ & 0.75 & $(0.48,1.15)$ & 0.19 & 0.81 & $(0.55,1.18)$ & 0.27 \\
\hline \multicolumn{7}{|l|}{ Renal cancers } \\
\hline \multicolumn{7}{|l|}{ Zoledronic acid 4 mg Q4W $(N=85)$} \\
\hline Denosumab 120 mg Q4W $(N=70)$ & 0.71 & $(0.43,1.17)$ & 0.18 & 0.75 & $(0.49,1.16)$ & 0.19 \\
\hline \multicolumn{7}{|l|}{ Lung cancer ${ }^{\mathrm{b}}$} \\
\hline \multicolumn{7}{|l|}{ Zoledronic acid 4 mg Q4W $(N=400)$} \\
\hline Denosumab 120 mg Q4W $(N=411)$ & 0.87 & $(0.68,1.11)$ & 0.26 & 0.90 & $(0.71 .1 .13)$ & 0.35 \\
\hline \multicolumn{7}{|l|}{ Non-small cell lung cancer ${ }^{\mathrm{c}}$} \\
\hline \multicolumn{7}{|l|}{ Zoledronic acid 4 mg Q4W $(N=352)$} \\
\hline Denosumab 120 mg Q4W $(N=350)$ & 0.85 & $(0.65,1.12)$ & 0.25 & 0.89 & $(0.69,1.15)$ & 0.38 \\
\hline \multicolumn{7}{|l|}{ Small cell lung cancer ${ }^{\mathrm{c}}$} \\
\hline \multicolumn{7}{|l|}{ Zoledronic acid 4 mg Q4W (N=48) } \\
\hline Denosumab 120 mg Q4W $(N=61)$ & 0.92 & $(0.47,1.80)$ & 0.80 & 0.91 & $(0.52,1.61)$ & 0.76 \\
\hline
\end{tabular}

Fig. 3 Kaplan-Meier estimates of a time to development of moderate or severe pain ( $>4$-point increase in Brief Pain Inventory Short Form [BPI-SF] among patients with baseline worst pain score $\leq 4)$ and $\mathbf{b}$ time to pain worsening ( $\geq 2$-point increase in BPI-SF among patients with baseline worst pain score $\leq 8$ )

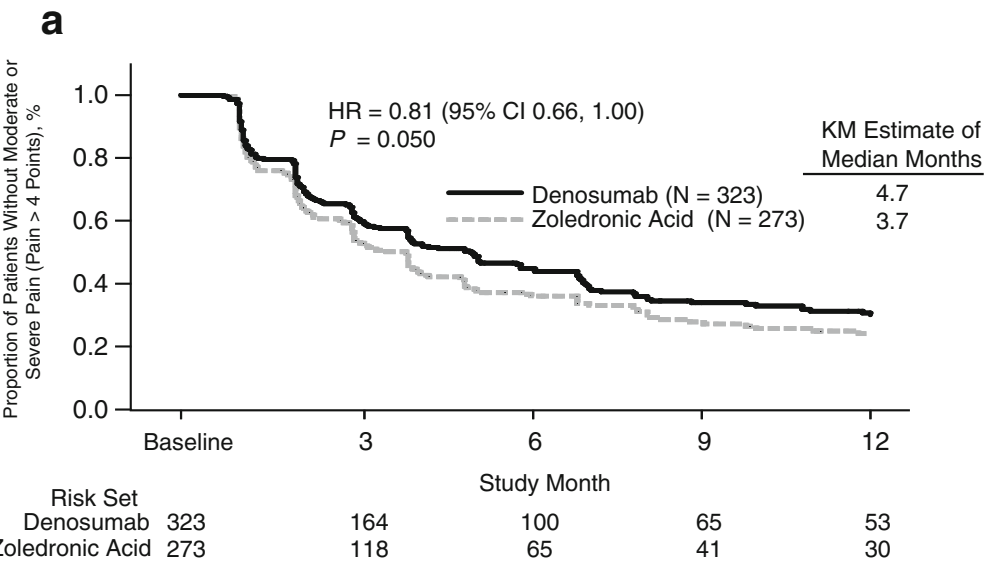

b

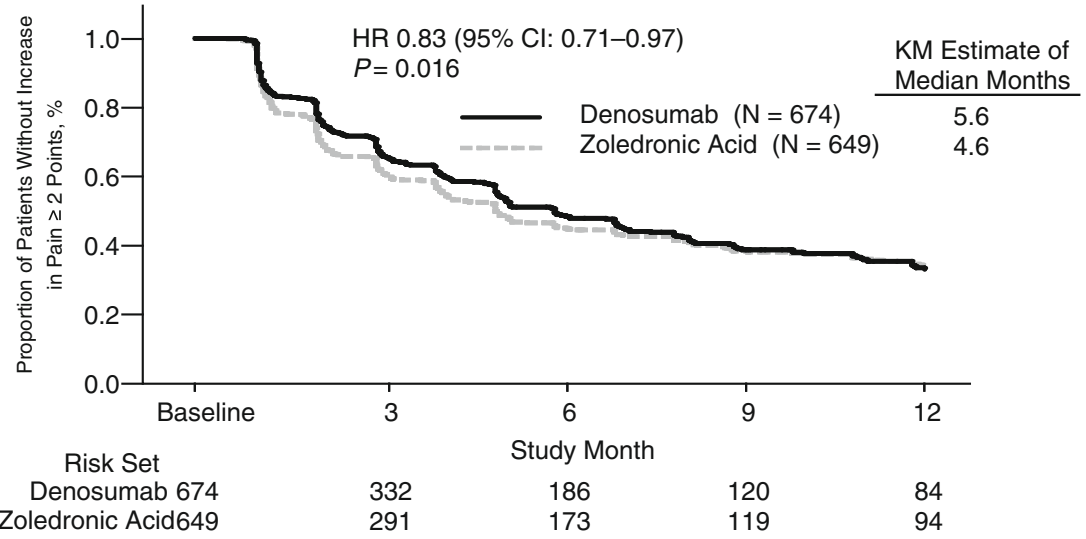


Table 3 Summary of adverse events

\begin{tabular}{|c|c|c|}
\hline Patient incidence, $n(\%)$ & $\begin{array}{l}\text { Denosumab } \\
(N=792)^{\mathrm{a}}\end{array}$ & $\begin{array}{l}\text { Zoledronic acid } \\
(N=786)^{\mathrm{a}}\end{array}$ \\
\hline \multicolumn{3}{|l|}{ Overall safety summary } \\
\hline Any adverse event & $757(96)$ & $751(96)$ \\
\hline \multicolumn{3}{|c|}{ Adverse events occurring with $\geq 20 \%$ frequency in either treatment group } \\
\hline Anemia & $219(28)$ & $250(32)$ \\
\hline Nausea & $219(28)$ & $233(30)$ \\
\hline Dyspnea & $210(26)$ & $181(23)$ \\
\hline Fatigue & $192(24)$ & $198(25)$ \\
\hline Constipation & $167(21)$ & $182(23)$ \\
\hline Anorexia & $151(19)$ & $182(23)$ \\
\hline Back pain & $149(19)$ & $173(22)$ \\
\hline Asthenia & $154(19)$ & $167(21)$ \\
\hline Vomiting & $164(21)$ & $166(21)$ \\
\hline Serious adverse events & $502(63)$ & $534(68)$ \\
\hline Deaths & $307(39)$ & $322(41)$ \\
\hline CTCAE grade 3 or 4 hypocalcemia & $35(4)$ & $14(2)$ \\
\hline Osteonecrosis of the jaw ${ }^{\mathrm{b}}$ & $6(0.8)$ & $9(1.1)$ \\
\hline Acute phase reaction ${ }^{c}$ & $56(7)$ & $116(15)$ \\
\hline Renal AEs ${ }^{\mathrm{d}}$ & $56(7)$ & $81(10)$ \\
\hline
\end{tabular}

${ }^{\mathrm{a}} N=$ number of patients who received at least one dose of investigational product

${ }^{\mathrm{b}} P=0.45$

${ }^{\mathrm{c}}$ Occurring within the first 3 days of treatment

${ }^{\mathrm{d}}$ Renal AEs were determined from a predefined list of preferred terms and included the following: increased blood creatinine, renal failure, acute renal failure, proteinuria, renal impairment, increased blood urea, anuria, oliguria, decreased urine output, azotemia, hypercreatininemia, chronic renal failure, and abnormal blood creatinine

metastatic breast or prostate cancer [13-15]. The difference in efficacy was seen early, at the third month of treatment, and KM curves continued to diverge throughout the study period.

A continuing benefit was evident with a reduction in the time to first-and-subsequent on-study SRE for denosumab compared with ZA. The relative risk reduction of $15 \%$ for any skeletal related event across the study was close to the overall relative risk reduction seen in breast and prostate cancers of 23 and $18 \%$, respectively [13,14], and is consistent with the relative reduction observed in bone turnover markers.

The distribution of types of first SREs was similar between patients receiving denosumab and ZA, although $3 \%$ fewer patients in the denosumab group experienced a first SRE of radiation to bone. Radiotherapy in this setting may have been given to treat bone pain or to prevent a fracture; however, information on specific indications for radiation treatment was not prospectively collected.

In addition, patients with skeletal metastases often develop bone pain in addition to other cancer-associated types of pain as a result of their disease. Pain is of acute importance in this population as it is often associated with declines in patient- reported QOL. In addition to preventing SREs, the prevention of pain is especially important for maintaining performance status in these patients. In advanced cancer patients with bone metastases, ZA treatment has been demonstrated to relieve pain $[8,22]$. In this analysis, denosumab further improved pain outcomes significantly, extending the time to severe pain by approximately 1 month compared with ZA for all patients. In the subset of patients with low pain scores at baseline, denosumab also significantly extended the time to pain worsening by approximately 1 month and the time to worsening of pain interference by more than 3 months. Denosumab and ZA had similar effects on time to worsening pain interference in the entire study population, and improvements in pain among patients who were already experiencing pain at baseline were also similar in the two treatment groups.

Similar to published observations in the parent trial [15] and in the two similarly designed trials in patients with breast and prostate cancer, respectively [13, 14], patients receiving denosumab in this solid tumor subgroup experienced a greater incidence of hypocalcemia events compared with those receiving ZA. This is consistent with our current understanding of the RANKL pathway and the higher potency of denosumab as an antiresorptive agent. Patients who experienced hypocalcemia generally had no clinical complications and were managed simply with calcium supplementation. It is highly recommended that all patients on bone-targeted therapy, whether with bisphosphonates or denosumab, receive supplemental calcium and vitamin D, with the exception of those with clinical hypercalcemia $[9,23]$.

Adjudicated incidence of ONJ was infrequent and not significantly different between treatment arms. Clinical characteristics of these ONJ cases and the oral interventions used to treat them were similar between treatment groups. The majority of patients with ONJ had a history of tooth extraction, poor oral hygiene, or dental appliance use.

Similar to the published registrational studies of ZA [8, 24], rates of AEs potentially associated with renal toxicity were elevated in patients treated with ZA and higher than those seen in patients treated with denosumab despite IV dosing adjustments for creatinine clearance as defined by the Zometa $^{\circledR}$ prescribing information [9]. This observation is particularly important in a population with advanced solid tumors, as these patients have a high prevalence of renal complications and a need for concomitant nephrotoxic therapy such as anti-neoplastic treatments or antibiotic therapy [25, 26]. Such renal complications can often limit the use of bisphosphonate therapy. Denosumab has no limitations with respect to renal function as it is a monoclonal antibody, which is eliminated by intracellular catabolism in phagocytes similar to the clearance mechanism of other therapeutic monoclonal antibodies, with no evidence of renal effects. Compared with $\mathrm{ZA}$, our analysis also found a reduced frequency of acute 
phase reactions with denosumab, which may provide greater tolerability to enable efficient administration on an outpatient basis.

Patients with solid tumors frequently have a shortened life expectancy, and in this subset analysis, the median time to first SRE in patients treated with denosumab was 21.4 months. Similar to previous trials of denosumab versus ZA in patients with advanced cancers [13-15], there was no difference in overall survival or investigator-assessed disease progression between treatment groups.

In conclusion, denosumab administered as a monthly subcutaneous injection represents a more efficacious treatment option compared with ZA for the management of bone metastases in this subset analysis of solid tumor patients. Potential additional advantages of denosumab treatment include reduced concerns surrounding the need for IV administration, dose adjustments for renal impairment, or concerns for acute-phase reactions.

Acknowledgments This study was funded by Amgen Inc. Editorial support for this article was provided by Jeffrey Coleman, MA, of Complete Healthcare Communications (Chadds Ford, PA), with funding from Amgen. The authors thank the patients and their families who contributed to this study. This study was funded by Amgen, Inc.

Conflicts of interest All authors had access to the primary data presented in this manuscript and will allow the editorial staff of Supportive Care in Cancer to review the data if necessary. G Smith and A Feng: employees and stockholders of Amgen. S Jun, R Dansey and $\mathrm{H}$ Yeh: former employees and current stockholders of Amgen. D Henry: speakers bureaus for Amgen and Ortho Biotech, and consultant for Watson Pharmaceuticals. S Vadhan-Raj: advisory boards and clinical trial research funding from Amgen. V Hirsh: advisory board for Amgen. $\mathrm{R}$ von Moos: speakers bureaus and unrestricted research grants from Roche and Amgen, and advisory boards for Roche, Amgen, and Novartis. V Hungria: No disclosures. L Costa: advisory boards for Amgen, Novartis, and Roche. PJ Woll: advisory board for Amgen.

\section{References}

1. Brown JE, Cook RJ, Major P, Lipton A, Saad F, Smith M, Lee KA, Zheng M, Hei YJ, Coleman RE (2005) Bone turnover markers as predictors of skeletal complications in prostate cancer, lung cancer, and other solid tumors. J Natl Cancer Inst 97:59-69

2. Coleman RE (2006) Clinical features of metastatic bone disease and risk of skeletal morbidity. Clin Cancer Res 12:6243s-6249s

3. Lipton A (2010) Implications of bone metastases and the benefits of bone-targeted therapy. Semin Oncol 37(Suppl 2):S15-S29

4. Portenoy RK, Lesage P (1999) Management of cancer pain. Lancet 353:1695-1700

5. Mavrogenis AF, Pala E, Romagnoli C, Romantini M, Calabro T, Ruggieri P (2012) Survival analysis of patients with femoral metastases. J Surg Oncol 105:135-141

6. Norgaard M, Jensen AO, Jacobsen JB, Cetin K, Fryzek JP, Sorensen HT (2010) Skeletal related events, bone metastasis and survival of prostate cancer: a population based cohort study in Denmark (1999 to 2007). J Urol 184:162-167
7. Saad F, Lipton A, Cook R, Chen YM, Smith M, Coleman R (2007) Pathologic fractures correlate with reduced survival in patients with malignant bone disease. Cancer 110:1860-1867

8. Rosen LS, Gordon D, Tchekmedyian NS, Yanagihara R, Hirsh V, Krzakowski M, Pawlicki M, De Souza P, Zheng M, Urbanowitz G, Reitsma D, Seaman J (2004) Long-term efficacy and safety of zoledronic acid in the treatment of skeletal metastases in patients with nonsmall cell lung carcinoma and other solid tumors: a randomized, phase III, double-blind, placebo-controlled trial. Cancer 100:2613-2621

9. Zometa ${ }^{\mathbb{R}}$ (Zoledronic acid) Package Insert. Novartis Pharmaceuticals Corporation, East Hanover, NJ. (2009).

10. Scott LJ, Muir VJ (2011) Denosumab: in the prevention of skeletalrelated events in patients with bone metastases from solid tumours. Drugs 71:1059-1069

11. Roodman GD (2004) Mechanisms of bone metastasis. N Engl J Med 350:1655-1664

12. Dougall WC, Chaisson M (2006) The RANK/RANKL/OPG triad in cancer-induced bone diseases. Cancer Metastasis Rev 25:541549

13. Stopeck AT, Lipton A, Body JJ, Steger GG, Tonkin K, de Boer RH, Lichinitser M, Fujiwara Y, Yardley DA, Viniegra M, Fan M, Jiang Q, Dansey R, Jun S, Braun A (2010) Denosumab compared with zoledronic acid for the treatment of bone metastases in patients with advanced breast cancer: a randomized, double-blind study. J Clin Oncol 28:5132-5139

14. Fizazi K, Carducci M, Smith M, Damiao R, Brown J, Karsh L, Milecki P, Shore N, Rader M, Wang H, Jiang Q, Tadros S, Dansey R, Goessl C (2011) Denosumab versus zoledronic acid for treatment of bone metastases in men with castration-resistant prostate cancer: a randomised, double-blind study. Lancet 377:813-822

15. Henry DH, Costa L, Goldwasser F, Hirsh V, Hungria V, Prausova J, Scagliotti GV, Sleeboom H, Spencer A, VadhanRaj S, von Moos R, Willenbacher W, Woll PJ, Wang J, Jiang Q, Jun S, Dansey R, Yeh H (2011) Randomized, double-blind study of denosumab versus zoledronic acid in the treatment of bone metastases in patients with advanced cancer (excluding breast and prostate cancer) or multiple myeloma. J Clin Oncol 29:1125-1132

16. Lipton A, Fizazi K, Stopeck AT, Henry DH, Brown JE, Yardley DA, Richardson GE, Siena S, Maroto P, Clemens M, Bilynskyy B, Charu V, Beuzeboc P, Rader M, Viniegra M, Saad F, Ke C, Braun A, Jun S (2012) Superiority of denosumab to zoledronic acid for prevention of skeletal-related events: a combined analysis of 3 pivotal, randomised, phase 3 trials. Eur J Cancer 48:3082-3092.

17. Kingsley LA, Fournier PG, Chirgwin JM, Guise TA (2007) Molecular biology of bone metastasis. Mol Cancer Ther 6:26092617

18. Cleeland CS (2006) The measurement of pain from metastatic bone disease: capturing the patient's experience. Clin Cancer Res 12: $6236 \mathrm{~s}-6242 \mathrm{~s}$

19. Chung K, Barlev A, Braun A, Qian Y, Zagari M. Development of the Analgesic Quantification Algorithm (AQA): a new scale to assess changes in analgesic use [abstract no. 3037]. Presented at: ECCO 15 and 34th ESMO Multidisciplinary Congress; Sept 20-24, 2009; Berlin, Germany.

20. Costa L, Fizazi K, Saad F, Brown JE, Von Moos R, Oudard S, Sternberg CN, Ganju V, Miller K, Wang H, Maniar T, Braun A (2013) Denosumab and zoledronic acid treatment in patients with genitourinary cancers and bone metastases [abstract no. 5079]. 2013 ACSO Annual Meeting. J Clin Oncol.

21. SERVICES USDOHAH (2010) Common Terminology Criteria for Adverse Events (CTCAE) Version 4.03. Available at: http://evs.nci. nih.gov/ftp1/CTCAE/CTCAE_4.03_2010-06-14_QuickReference_ 8.5x11.pdf. Accessed 5 June 2013. 
22. Weinfurt KP, Anstrom KJ, Castel LD, Schulman KA, Saad F (2006) Effect of zoledronic acid on pain associated with bone metastasis in patients with prostate cancer. Ann Oncol 17:986-989

23. XGEVA ${ }^{\circledR}$ (Denosumab) Package Insert. Amgen Inc., Thousand Oaks, CA. (2010)

24. Rosen LS, Gordon D, Kaminski M, Howell A, Belch A, Mackey J, Apffelstaedt J, Hussein MA, Coleman RE, Reitsma DJ, Chen BL, Seaman JJ (2003) Long-term efficacy and safety of zoledronic acid compared with pamidronate disodium in the treatment of skeletal complications in patients with advanced multiple myeloma or breast carcinoma: a randomized, double-blind, multicenter, comparative trial. Cancer 98:1735-1744

25. Kleber M, Cybulla M, Bauchmuller K, Ihorst G, Koch B, Engelhardt M (2007) Monitoring of renal function in cancer patients: an ongoing challenge for clinical practice. Ann Oncol 18:950-958

26. Launay-Vacher V, Gligorov J, Le Tourneau C, Janus N, Spano JP, Ray-Coquard I, Oudard S, Pourrat X, Morere JF, Deray G, Beuzeboc P (2010) Prevalence of renal insufficiency in breast cancer patients and related pharmacological issues. Breast Cancer Res Treat 124: $745-753$ 